\title{
Interaktive Internetplattform der Herzstiftung
}

\author{
A. Hoffmann ${ }^{a}$, H. Gohlke, \\ W. Bachmann', \\ U. Blessbergerc, \\ Ch. Bächtold ${ }^{a}$,T. Junker ${ }^{a}$
}

\section{a Schweizerische Herzstiftung \\ b Mitglied des Vorstandes Deutsche Herzstiftung e.V. \\ c VR Consult AG}

\section{Hintergrund und Projektbeschrieb}

Die Schweizerische Herzstiftung wurde im Jahr 1967 von einer Gruppe weitsichtiger Kardiologen gegründet. Sie verfolgt drei Zweckbestimmungen: Forschungsförderung, Information und Beratung von Betroffenen und Angehörigen sowie Aufklärung und Prävention in der Bevölkerung. Während die Spendengelder zur Hauptsache der Forschungsförderung zufliessen, werden die übrigen Projekte auch aus zweckbestimmten Förder- und Sponsorengeldern finanziert. Die Stiftung ist seit 1989 ZEWO-zertifiziert.

Im September 2009 wurde ein Konzept für eine Plattform zur Förderung der Patientenaufklärung und Compliance erarbeitet. Dieses Konzept beinhaltet auch die Entwicklung einer für Laien und Fachpersonen gleichermassen zugänglichen InternetPlattform, über die eine individualisierte kardiovaskuläre Risikoabschätzung mit der Möglichkeit einer interaktiven Optimierung des Gesundheitsverhaltens angeboten wird.

Das Projekt gliedert sich in mehrere Phasen, von denen die erste seit September 2013 im Internet unter www.swissheart-coach.ch zugänglich ist. Es handelt sich um einen Risikorechner, der in erster Linie die relative Erhöhung des kardiovaskulären Erkrankungsrisikos im Vergleich zu einer gleichaltrigen Person ohne veränderbare bzw. mit optimierten Risikofaktoren darstellt. Im Programm kann ein daraus resultierendes Potential von sogenannten Gesundheitsfaktoren durch Auswahl vorgeschlagener Verhaltensänderungen in variablem Mass interaktiv ausgeschöpft werden. Daten werden in dieser Phase nicht gespeichert, jedoch wird am Schluss einer Sitzung ein anonymer gedruckter Report mit detaillierten Empfehlungen generiert. Ergänzend werden Angebote für weiterführende Beratungen gemacht, einschliesslich der Telefon- und Internetberatung durch Ärzte der Herzstiftung. Schliesslich erscheinen zahlreiche Links zu existierenden Informationsmitteln und Unterstützungsangeboten und zur Wissensdatenbank ELIPS des Universitätsspitals Genf. Auf einer speziell für Fachpersonen konzipierten Seite finden sich Quellenangaben zu den wissenschaftlichen Berechnungen.

\section{Methodik der Risikoberechnung}

Zur Ermittlung des absoluten Risikos legt der Swissheart-Coach den AGLA Score zugrunde. Wie der ESC-Score berücksichtigt der AGLA Score gegenüber PROCAM die Resultate der MONICA-Studie, gemäss

\section{Zusammenfassung}

Die Schweizerische Herzstiftung hat sich in Zusammenarbeit mit Experten und Vertretern medizinischer Fachgesellschaften die Lancierung eines interaktiven webbasierten kardiovaskulären Schulungsund Coaching-Programms zum Ziel gesetzt. Mit dem Programm www.swissheart-coach.ch sollen gesundheitsbewusste Personen und Patienten unterstützt werden im Umgang mit kardiovaskulären Risikofaktoren bei Prävention und Bewältigung von Krankheit. Auch für Fachpersonen kann das Programm eine Hilfe bei der Begleitung und Beratung von Betroffenen sein. Der «Swissheart-Coach» ist einzigartig, indem er sämtliche bekannte Risikofaktoren einbezieht. Dabei wird auf acht sogenannte Gesundheitsfaktoren fokussiert: Nichtrauchen, körperliche Aktivität, Ernährung, Körpergewicht, Stressmanagement, Kontrolle von Blutdruck, Lipid- und Glukosestoffwechsel. Im «Swissheart-Coach» ist ein Risikorechner integriert, der auf Basis wissenschaftlicher Evidenz das absolute Risiko gemäss AGLA Score, das berechnete «Herzalter» und das relative Risiko im Vergleich zu einer Person mit optimiertem Gesundheitsverhalten verständlich darstellt. Mit einer Vielzahl wählbarer Massnahmen wird die Veränderung direkt sichtbar gemacht. Ein detaillierter individueller Bericht kann ausgedruckt werden. Ergänzt wird das Programm durch zahlreiche weiterführende Links, Informationsquellen und Hinweise auf medizinische Beratungsmöglichkeiten.

der die Schweiz innerhalb Europas ein NiedrigrisikoLand ist, mit einem Gewichtungsfaktor von 0,7 [1]*. Sodann wird für die Berechnung des relativen Risikos die prognostische Bedeutung des BMI, der Anzahl der gerauchten Zigaretten (statt bei Rauchen einfach nur ja oder nein anzugeben) und der körperlichen Aktivität anhand der in multivariaten Regressionsanalysen erhobenen unabhängigen Bedeutung dieser Parameter herangezogen [2, 3a, 3b]. 


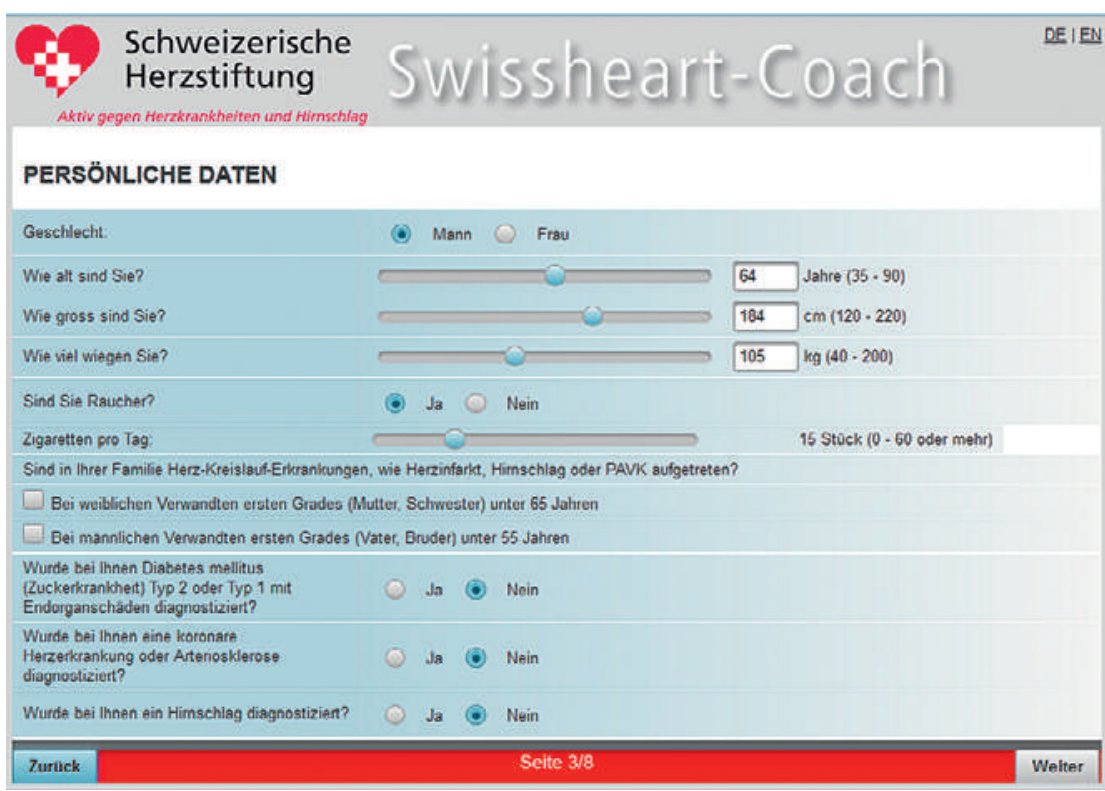

Abbildung 1

Beispiel einer Eingabemaske für persönliche Daten.

\section{Darstellung aller einbezogenen Variablen}

Folgende Variablen werden für die Risikostratifizierung beziehungsweise Risikoqualifizierung verwendet: Alter, Geschlecht, systolischer Blutdruck, Gesamtcholesterin, LDL-Cholesterin, HDL-Cholesterin, Triglyzeride entsprechend den ESC-Prevention Guidelines [4], Body-Mass-Index, Anzahl der täglich gerauchten Zigaretten und körperliche Aktivität berechnet als Kalorienverbrauch pro Woche $[5,2]$ auf der Basis metabolischer Äquivalente (MET).

Die Ernährung wird auf ihre graduelle Übereinstimmung zur mediterranen Kost nach dem alternativen mediterranen Score beurteilt [6]. Der Faktor Stress wird entsprechend der Interheart-Studie in die Risikobeurteilung miteinbezogen [7]. Bei mehr als drei Risikofaktoren wird eine adjustierte Gewichtung vorgenommen.

\section{Verfahren bei «missing data»}

Da nicht davon ausgegangen werden kann, dass sämtliche Anwender alle gewünschten Daten zur Verfügung stellen können, mussten Verfahren gefunden werden, wie mit fehlenden Daten umzugehen ist. Falls dem Anwender seine Daten zum Blutdruck und den Blutfetten nicht bekannt sind, werden vom System obere nicht pathologische Werte angenommen und es folgt der Hinweis auf die eingeschränkte Aussagekraft der Berechnung sowie die Aufforderung, die nicht bekannten Daten im Rahmen eines Besuchs beim Hausarzt oder in der Apotheke bestimmen zu lassen.

\section{Laiengerechte Darstellung der Resultate}

Die in der medizinischen Fachwelt vorhandenen Risikorechner sind für die Anwendung durch Experten konzipiert. Daher wurde in der Entwicklung des
Programms grösster Wert darauf gelegt, das Programm laiengerecht zu gestalten. Es wird zunächst eine Kategorisierung des absoluten kardiovaskulären Risikos in die Stufen niedrig, mittel, hoch und sehr hoch vorgenommen. Im Weiteren erfährt der Anwender sein rechnerisches Herzalter. Schliesslich wird das relative Risiko berechnet. Dabei handelt es sich um einen Vergleich des Anwenders zu einer Person mit optimalem Gesundheitsverhalten. Die jeweilige Ausprägung der acht dafür berücksichtigten Gesundheitsfaktoren wird durch Ampelfarben in einer Grafik quantitativ dargestellt. Die Interventionen und die damit verbundenen Veränderungen werden ebenfalls grafisch umgesetzt.

\section{Motivation zur Mobilisierung eigener Ressourcen}

Die besondere Stärke des «Swissheart-Coach» liegt darin, dass dieses Programm den Dialog mit dem Anwender aufnimmt und ihn motiviert, seine gesundheitlichen Ressourcen zu aktivieren. Den Benutzern wird eine breite Palette von möglichen Verhaltensweisen zur Auswahl angeboten, die jeweils motivierend quittiert werden. In einer zweiten Projektphase ab 2014 soll es möglich sein, die individuellen Daten $\mathrm{zu}$ verschiedenen Zeitpunkten einzugeben, zu speichern und eine durch die Benutzer wählbare Recall-

Mitglieder der Projektgruppe und Repräsentanten der involvierten Fachgesellschaften

Andreas Hoffmann, Facharzt für Kardiologie, Mitglied Stiftungsratsaussschuss Schweizerische Herzstiftung, Projektleiter

Christa Bächtold, Bereichsleiterin Patienten, Schweizerische Herzstiftung

Edouard Battegay, Chefarzt Innere Medizin USZ, Vorstand SGIM

Thomas Berger, Klin. Psychologe, Universität Bern Baris Gencer, ELIPS HCUGE

Helmut Gohlke, Facharzt für Kardiologie,

Vorstandsmitglied Deutsche Herzstiftung

Ueli Grüninger, Geschäftsführer KHM

Gudrun Haager, Sportwissenschafterin, KS St. Gallen René Lerch, Facharzt für Kardiologie,

Mitglied Stiftungsrat Schweizerische Herzstiftung Katharina Meyer, Leistungsphysiologin, Inselspital Bern Nicolas Rodondi, Leiter Poliklinik, Chefarzt Klinik für Allg. Innere Medizin Inselspital Bern, Ausschussmitglied AGLA Andreas Zeller, Institut für Hausarztmedizin Uni Basel, Vertreter SGAM

\section{Vertreter der Industriepartner}

Edith Helmle, AstraZeneca

Pascal Koepfli, AstraZeneca

\section{Projekt-Realisation}

Wolfgang Bachmann, Projektleiter VR Consult AG, D-Niedernberg

Uwe Blessberger, Technischer Projektleiter, VR Consult AG

Projekt-Controlling

Therese Junker, Geschäftsführerin Schweizerische Herzstiftung 


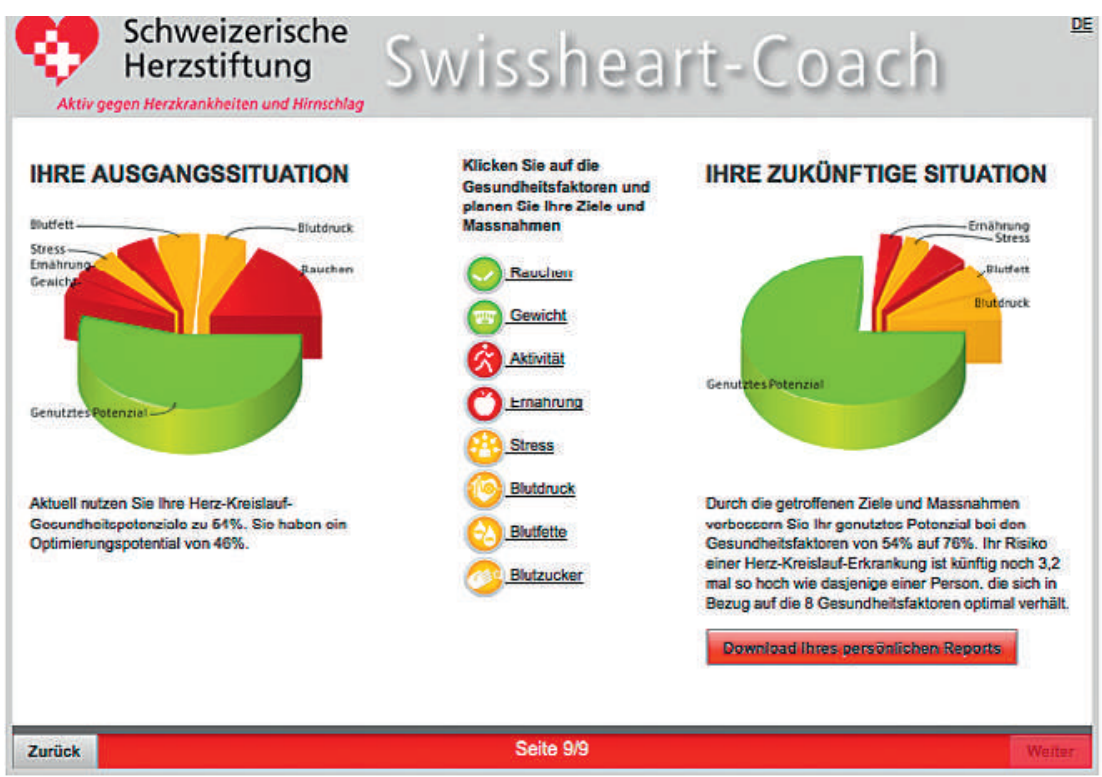

Abbildung 2

Screenshot einer Resultatseite. halten Unterstützung durch vertiefende Informationen und Hinweise auf Fortbildungsangebote.

\section{Diskussion und Ausblick}

Mit dem beschriebenen Vorgehen betritt die Schweizerische Herzstiftung in vielerlei Hinsicht Neuland. Es werden Methoden zur Berechnung des kardiovaskulären Risikos angewendet, die zwar mit den herkömmlichen Risikomodellen vergleichbar sind und zum Teil auch auf ähnlichen Daten beruht. Neu ist aber eine stärkere Differenzierung einzelner Variablen wie beispielsweise Ernährungs- und Bewegungsgewohnheiten, die quantitative Berücksichtigung des Nikotinkonsums sowie der Einbezug von psychosozialen Faktoren. Die Darstellung des berechneten Risikos erfolgt in erster Linie als relative Erhöhung im Vergleich mit einer Person, die alle veränderbaren Parameter optimiert und somit kein Veränderungspotential hat. Das individuell vorhandene Veränderungspotential wird quantitativ dargestellt in Abhängigkeit der vom Benutzer gewählten Massnahmen.

Zwar kann das relative Risiko einer Person unter dem Aspekt des absoluten Risikos unterschiedlich gewichtet werden, jedoch ist die Mobilisation von Gesundheitsressourcen zur Beeinflussung des relativen Risikos auch bei niedrigem Ausgangsrisiko wichtig, wenn man bei diesen meist jungen Personen den Aspekt des «lifetime risk» berücksichtigt.

Ärztinnen und Ärzte werden in ihren Praxen früher oder später mit den Resultaten beziehungsweise den gedruckten Berichten des «Swissheart-Coach» konfrontiert werden oder möchten ihre Patienten auf dieses Angebot hinweisen. Zur Unterstützung der Fachpersonen wird die Schweizerische Herzstiftung das Programm an Tagungen und in Fortbildungsseminaren demonstrieren. Zudem wurde ein Standardbrief für die behandelnden Ärzte zum Download bereitgestellt.

Die Frage des Effekts des Angebotes bedarf einer sorgfältigen wissenschaftlichen Begleitung. Geplant ist eine Kohortenstudie über einen definierten Zeitraum anhand einer Stichprobe von anonymisierten Nutzern in der Phase II des Projekts im Vergleich zu einem Kontrollkollektiv vor der Aufschaltung. 


\section{Literatur}

1 Riesen WF, Darioli F, Noseda G, Bertel O, Buser P. Empfehlungen zur Prävention der Atherosklerose. Schweiz Ärztezeitung. 2005;86(22):1355-61.

2 Gohlke H, Winter M, Karoff M, Held K. CARRISMA a new tool to improve risk stratification and guidance of patients in cardiovascular risk management in primary prevention. Eur J Cardiovasc Prev Rehabil. 2007;14:141-8.

3a Gohlke H, Albus C, Bönner G, Darius H, Eckert S, Gohlke-Bärwolf et al. Empfehlungen der Projektgruppe Prävention der DGK zur risikoadjustierten Prävention von Herz- und Kreislauferkrankungen. Teil 1: Risikostratifikation und Umsetzung der Prävention. Kardiologe. 2012;6:63-76.

3b Gohlke H, Albus C, Bönner G, Darius H, Eckert S, Gohlke-Bärwolf C, et al. Empfehlungen der Projektgruppe Prävention der DGK zur risikoadjustierten Prävention von Herz- und Kreislauferkrankungen. Teil 2: Lebensstil und kardiovaskuläres Risiko. Kardiologe. 2012;6:249-62.
4 Perk J, De Backer G, Gohlke H, Graham I et al. European Guidelines on Cardiovascular Disease Prevention in Clinical Practice European Heart Journal. 2012;33:1635-701.

5 Teo KK, Ounpuu S, Hawken S, Pandey M, Valentin V, Hunt D (on behalf of the INTERHEART Study Investigators). Tobacco use and risk of myocardial infarction in 52 countries in the INTERHEART study: a case-control study. Lancet. 2006;368:647-58.

6 Mitrou PN, Kipnis V, Thiébaut ACM, Reedy J, Subar AF, Wirfält E, et al. Mediterranean Dietary Pattern and Prediction of All-Cause Mortality in a US Population Results From the NIH-AARP Diet and Health Study. Arch Intern Med. 2007;167:2461-8.

7 Yusuf S, Hawken S, Ôunpuu S, Dans T, Avezum A, Lanas, F (on behalf of the INTERHEART Study Investigators). Effect of potentially modifiable risk factors associated with myocardial infarction in 52 countries (the INTERHEART study): case-control study. Lancet. 2004;364:93752. 